\title{
A Study on W Ursae Majoris-Type Systems Recognised by the ROTSE-IIId Experiment
}

\author{
D. Çoker ${ }^{1,5}$, S. Özdemir ${ }^{1}$, C. Yeşilyaprak ${ }^{2}$, S. K. Yerli ${ }^{3}$, N. Aksaker ${ }^{4}$ and B. B. Güçsav ${ }^{1}$ \\ ${ }^{1}$ Faculty of Science, Department of Astronomy and Space Sciences, Ankara University, Ankara, Turkey \\ ${ }^{2}$ Faculty of Science, Department of Physics, Atatürk University, Erzurum, Turkey \\ ${ }^{3}$ Department of Physics, Middle East Technical University, Ankara, Turkey \\ ${ }^{4}$ Çukurova University, Vocational School of Technical Sciences, Adana, Turkey \\ ${ }^{5}$ Corresponding author. Email: denizcoker@gmail.com
}

(Received June 6, 2012; Accepted August 28, 2012; Online Publication January 24, 2013)

\begin{abstract}
:
We present a study on characterising the light curves of W UMa-type systems gathered from the archive containing 5 years of data observed with the Robotic Optical Transient Search Experiment Telescope (ROTSE-IIId) located in Turkey. A sample of $45 \mathrm{~W}$ UMa-type systems was studied on the basis of Fourier decomposition of light curves and some basic geometrical parameters, namely degree of contact $(f)$, mass ratio $(q)$, and orbital inclination $(i)$, as approximated values for these systems were determined. Moreover, methods based on the Fourier transform technique were applied to the discrete data to determine the orbital periods of those systems. Preliminary estimates for the system parameters were presented and compared with the values available in the literature.
\end{abstract}

Keywords: binaries: eclipsing - methods: data analysis

\section{INTRODUCTION}

W UMa-type stars belong to the class of contact binaries and can be briefly characterised by (i) continuous light variations with amplitudes less than $\sim 0.9$ mag, (ii) orbital periods shorter than $24 \mathrm{~h}$, (iii) almost equal depths of minima, and (iv) usual spectral types between $\mathrm{F}$ and $\mathrm{K}$. The common envelope structure of W UMa stars was first introduced by Lucy (1968). Physical structures and evolutionary stages of W UMa systems were discussed in detail by various authors (Binnendijk 1970, 1977; Eggleton 1996; Kähler 2004; Li et al. 2008).

Wide-angle sky surveys such as the Optical Gravitational Lensing Experiment (OGLE; Udalski et al. 1992, 1994), the Robotic Optical Transient Search Experiment Telescope (ROTSE; Akerlof et al. 2003) projects, as well as data from orbiting experiments, i.e. Kepler Mission (Borucki et al. 2010), the Optical Monitoring Camera (OMC) onboard the INTEGRAL satellite (Mas-Hesse et al. 2004) have extremely increased the photometric data to be evaluated to uncover the variability types and periodicities of the stellar objects. A total of 45 eclipsing binaries known as W UMa-type in the literature were selected from the database of the ROTSE-IIId telescope maintained by the TÜBİTAK National Observatory (TUG) in Turkey. The periodicity of unevenly sampled data for each system was determined by the Lomb-Scargle (L-S) algorithm (Lomb 1976; Scargle 1982) and used to construct folded light curves. Then, a classification technique, which was also successfully applied to Hipparcos light curves by Selam (2004), based on the Fourier decomposition of light curves in cosine series (Rucinski 1993), was performed to obtain preliminary parameters of the systems. Approximate values of the degree of contact $(f)$, mass ratio $(q)$, and orbital inclination $(i)$ of each system, together with periodicities and Fourier coefficients, are presented in this study.

We aim to extend this work to automatically recognise all W UMa-type systems in the full dataset of the ROTSEIIId experiment. However, note that the distorting effects in the light curves, namely, the O'Connell effect, asymmetries because of spot activity, third light contamination or observational scattering, can strongly restrict the reliability of this classification method.

\section{OBSERVATIONS AND DATA ANALYSIS}

\subsection{Observations}

The observational data used in this study were provided by the Turkish part of the public records of the ROTSE-IIId 
telescope's archival data. The ROTSE-III telescopes are located at four different regions around the world and they are dedicated to observe optical afterglows of gamma-ray bursts. One of these telescopes, ROTSE-IIId, is located at TUG in Antalya, Turkey. It has an aperture of $45 \mathrm{~cm}$ and a $2048 \times 2048$ CCD with the pixel scale of 3.3 arcsec pixel ${ }^{-1}$ for a total field of view of $1^{\circ} .85 \times 1^{\circ} .85$. It uses no filters but have a wide passband that peaks at $550 \mathrm{~nm}$. The ROTSE-III telescopes were described in detail by Akerlof et al. (2003).

The Turkish side of the ROTSE-IIId archive consists of 422 different sky pointings with more than a quarter million of CCD frames taken from these pointings. Due to ROTSEIIId's observational limitations, each night, only 1-20 frames of individual pointings were observed with 60,20 , and mostly $5 \mathrm{~s}$ of exposure times. A total of $118 \mathrm{~W}$ UMa-type variables examined in this study were retrieved from a SIMBAD query which fell into only 48 different pointings in this archive.

\subsection{Data Analysis}

The ROTSE-IIId telescope is equipped with an automated data reduction pipeline. Dark frames that are accumulated each night are used in the data reduction pipeline, together with a proper sky-flat and fringe frame. For detection and in order to obtain instrumental magnitudes of the detected objects, aperture photometry is done with a 5-pixel (17 arcsec) diameter aperture by use of the SEXTRACTOR code (Bertin \& Arnouts 1996). These magnitudes are calibrated by comparing all field stars against the USNO A2.0 $R$-band magnitudes (Monet 1998).

According to the pointing, 162-2 238 frames were used in generating one light curve for each system. The outcome from the above analysis was a total of 118 light curves of wellknown W UMa stars, of which 45 candidates had enough number of data points in their light curves for any period analysis in detail. Two of the systems, Cl* NGC 6791 KR V118 and V791 Cep were also excluded since they were 17.68 and $17.27 \mathrm{mag}$, respectively, which are $\sim 1.0 \mathrm{mag}$ fainter than the limiting magnitude of ROTSE-IIId (Güçsav et al. 2012). Times of all systems used in this study were corrected to the Solar System barycenter.

The L-S technique (Lomb 1976; Scargle 1982) was applied to discretely sampled data to obtain any meaningful periodicity. Periods were determined by a script, written based on the L-S algorithm, and the results were compared with the values given in the literature. Of the 43 stars we analyzed, 37 had periods consistent with those in the literature. However, our results point out different periods for the V455 Mon, XX Lyn, and V981 Cyg systems, which will be discussed in Section 3. Period determination has failed for the systems USNOA2.00900-11608642, 2MASS J19033572+4336124, and 2MASS J20292467+6029444 when the L-S algorithm was applied. This was probably caused by fewer sampled data points or poor data quality of the light curves. Hence, these three systems were excluded in the Fourier series fitting step.
Rucinski $(1973,1993)$ showed that light curves of W UMatype systems strongly depend on geometrical parameters such as degree of contact $f$, orbital inclination $i$, and mass ratio $q$ and that they can be represented well by a low-order cosine series, $l(\theta)=\sum_{i=0}^{6} a_{i} \cos (2 \pi \mathrm{i} \theta)$, where $l(\theta), a_{i}$, and $\theta$ are normalised light in light units, Fourier coefficients, and angular phase of the system in radians, respectively. To evaluate the values of $f, i$, and $q$, tables of Fourier coefficients listed in Rucinski's personal Web site ${ }^{1}$ were used after modifying light curves as suggested by Rucinski (1993), by normalising to unity and shifting the primary, i.e. deeper, minimum to zero phase. Rucinski (1993) also computed the model light curves by adopting a representative solar case for the photometric band $V$. However, since the instrumental response curve of ROTSE-IIId has a central wavelength at $550 \mathrm{~nm}$, any transformation to our data was not performed in our study. Fourier coefficients were first used to determine the degree of contact $f$ for each system with steps of $\Delta f=0.1$. Then, using the Fourier coefficients of $a_{1}, a_{2}$, and $a_{4}$, corresponding geometrical parameters were tried to be determined for 40 W UMa systems.

\section{RESULTS AND DISCUSSION}

The main aim of this study is to recognise the W UMa-type binaries selected from the archival database of the ROTSEIIId telescope and to obtain preliminary system parameters by fitting truncated Fourier series on the light curves. To achieve this aim, orbital periods were primarily calculated from discretely sampled data by an L-S-based algorithm and the results were compared with values in the literature. The periodicities on the basis of the L-S algorithm for $43 \mathrm{~W}$ UMa systems are presented in Table 1. Three of the systems, V455 Mon, XX Lyn, and V981 Cyg, produced periodicities different from those given in the literature. However, our results seem to be much more plausible since (i) false-alarm probability is very low, (ii) not only the L-S algorithm but ANOVA (Schwarzenberg-Czerny 1996) and CLEANest (Foster 1995) algorithms also point out the same periods, and (iii) phase coverage and quality of the data are very satisfying for these three systems. The main reason for the rejection of three systems, USNO-A2.00900-11608642, 2MASS $\mathrm{J} 19033572+4336124$, and 2MASS J20292467+6029444, in the Fourier analysis is that the first two systems have fewer than 100 data points spread over a time span of 4 months and the third system has a brightness of $16.20 \mathrm{mag}$, which was pointed out earlier as the limiting magnitude of ROTSE-IIId. Hence, the light curves of these systems are extremely scattered or it is impossible to obtain any reasonable periodicities. Note also that the time span of the observations, i.e. 5 years, is too long when variation intervals in the light curves and periods of W UMa-type systems are considered (Karimie 1983; Derman, Demircan, \& Selam 1991; Awadalla 1988, 1994).

\footnotetext{
${ }^{1}$ http://www.astro.utoronto.ca/ rucinski/tables/coef
} 
Table 1. Selected W UMa-Type Binaries from the ROTSE-IIId Database with Calculated Periods

\begin{tabular}{|c|c|c|c|c|c|c|c|c|}
\hline Name & $\begin{array}{c}\alpha_{2000} \\
(\mathrm{~h}: \mathrm{m}: \mathrm{s})\end{array}$ & $\begin{array}{c}\delta_{2000} \\
(\mathrm{~d}: \mathrm{m}: \mathrm{s})\end{array}$ & $V(\mathrm{mag})$ & $\begin{array}{c}\text { No. } \\
\text { of Frames }\end{array}$ & $\begin{array}{l}\text { Period (d) } \\
\text { (This Paper) }\end{array}$ & $\begin{array}{l}\text { False-Alarm } \\
\text { Probability }\end{array}$ & $\begin{array}{c}\text { Period (d) } \\
\text { (Literature) }\end{array}$ & Ref. $^{a}$ \\
\hline V959 Cas & 001202.65 & +550519.5 & $11.90(\mathrm{R})$ & 302 & 1.064930 & $6.13 \mathrm{E}-49$ & 1.065200 & 6 \\
\hline V472 And & 001550.06 & +412803.1 & $13.10(\mathrm{R})$ & 484 & 0.850631 & $1.70 \mathrm{E}-48$ & 0.850560 & 16 \\
\hline V473 And & 001605.37 & +415124.2 & $13.63(\mathrm{R})$ & 442 & 0.401308 & $4.56 \mathrm{E}-52$ & 0.401360 & 16 \\
\hline V755 Cep & 001824.20 & +730724.0 & 12.99 & 562 & 0.392168 & $1.98 \mathrm{E}-75$ & 0.392170 & 6 \\
\hline V490 And & 002648.75 & +415004.2 & 14.60 & 598 & 0.424173 & $1.34 \mathrm{E}-41$ & 0.424180 & 6 \\
\hline ES Cep & 005049.89 & +851611.7 & 15.87 & 1048 & 0.342448 & $1.11 \mathrm{E}-55$ & 0.342457 & 8 \\
\hline V790 Cep & 010150.42 & +852400.2 & 12.80 & 1187 & 0.321229 & $9.42 \mathrm{E}-88$ & 0.321496 & 8 \\
\hline V793 Cep & 010831.75 & +851254.2 & 16.35 & 869 & 0.306730 & $5.20 \mathrm{E}-18$ & 0.307213 & 8 \\
\hline GR Psc & 010931.92 & +223919.4 & 11.26 & 259 & 0.494354 & $8.05 \mathrm{E}-37$ & 0.494339 & 1 \\
\hline V1107 Cas & 012314.59 & +613452.9 & $12.80(\mathrm{R})$ & 344 & 0.273409 & $9.47 \mathrm{E}-44$ & 0.273406 & 6 \\
\hline DN Cam & 044246.24 & +725841.9 & 8.56 & 187 & 0.498306 & $1.77 \mathrm{E}-22$ & 0.498309 & 9 \\
\hline V415 Cam & 055738.19 & +8038 18.4 & $13.60(\mathrm{R})$ & 364 & 0.432606 & $2.72 \mathrm{E}-56$ & 0.432620 & 15 \\
\hline AH Gem & 065317.90 & +155923.0 & 14.40 & 149 & 0.336780 & $2.11 \mathrm{E}-16$ & 0.336752 & 7 \\
\hline V455 Mon & 065500.88 & +000719.3 & $14.00(\mathrm{R})$ & 178 & 0.507955 & $4.09 \mathrm{E}-23$ & 0.681621 & 6 \\
\hline $\mathrm{CZ}$ CMi & 071657.33 & +09 1235.4 & 10.54 & 194 & 0.426384 & $4.74 \mathrm{E}-28$ & 0.426388 & 6 \\
\hline XX Lyn & 074230.02 & +414057.2 & $15.10(\mathrm{R})$ & 241 & 0.313527 & $1.73 \mathrm{E}-28$ & 0.371987 & 18 \\
\hline $\mathrm{AH} \mathrm{Cnc}$ & 085137.85 & +115057.1 & 13.33 & 698 & 0.360458 & $3.01 \mathrm{E}-95$ & 0.360441 & 6 \\
\hline RT LMi & 094948.32 & +342715.4 & $10.99(\mathrm{R})$ & 179 & 0.374883 & $3.74 \mathrm{E}-29$ & 0.374918 & 6,12 \\
\hline 2MASS J11403001+7111021 & 114030.02 & +711102.2 & 13.76 & 794 & 0.434929 & $2.20 \mathrm{E}-135$ & 0.434911 & 10 \\
\hline 2MASS J11483649+7107507 & 114836.53 & +710750.8 & 14.25 & 786 & 0.376846 & $1.16 \mathrm{E}-123$ & 0.376832 & 10 \\
\hline GQ Boo & 145936.67 & +250244.8 & $12.70(\mathrm{R})$ & 368 & 0.384634 & $2.08 \mathrm{E}-42$ & 0.384657 & 4 \\
\hline GR Boo & 145954.55 & +255433.6 & 11.51 & 373 & 0.376676 & $7.52 \mathrm{E}-48$ & 0.376664 & 4 \\
\hline V829 Her & 165547.88 & +351058.0 & 10.39 & 1818 & 0.358156 & $8.95 \mathrm{E}-221$ & 0.358157 & 17 \\
\hline V2203 Oph & 174943.28 & +042824.3 & 10.85 & 920 & 0.454994 & $2.20 \mathrm{E}-117$ & 0.455005 & 1 \\
\hline USNO-A2.00900-11608642 & 180651.41 & +050930.2 & 15.00 & 96 & - & - & 0.478173 & 13 \\
\hline V591 Lyr & 182436.82 & +381734.0 & $12.20(\mathrm{R})$ & 259 & 0.300356 & $9.07 \mathrm{E}-41$ & 0.300318 & 4 \\
\hline V574 Lyr & 182712.20 & +361436.2 & 12.29 & 111 & 0.273125 & $8.78 \mathrm{E}-12$ & 0.273127 & 1 \\
\hline 2MASS J18413909-0044433 & 184139.09 & -004443.3 & 11.87 & 74 & 0.335939 & $5.34 \mathrm{E}-08$ & 0.335915 & 1 \\
\hline 2MASS J18562000+4556486 & 185620.01 & +455648.7 & 14.63 & 178 & 0.349949 & $1.16 \mathrm{E}-08$ & 0.349951 & 3 \\
\hline 2MASS J19033572+4336124 & 190335.73 & +433612.4 & 13.93 & 53 & - & - & 0.303654 & 2 \\
\hline 2MASS J19062202+4338096 & 190622.02 & +433809.7 & 13.79 & 492 & 0.374355 & $4.36 \mathrm{E}-82$ & 0.374351 & 2 \\
\hline ROTSE1 J190830.01+433601.5 & 190830.02 & +433601.6 & 11.56 & 490 & 0.392762 & $4.06 \mathrm{E}-79$ & 0.392740 & 4 \\
\hline 2MASS J19180543+4441153 & 191805.44 & +444115.3 & 12.91 & 473 & 0.608284 & $9.28 \mathrm{E}-70$ & 0.608121 & 4 \\
\hline V2364 Cyg & 192211.74 & +492834.4 & 11.26 & 347 & 0.592121 & $2.97 \mathrm{E}-52$ & 0.592053 & 4 \\
\hline V981 Cyg & 194503.57 & +562450.8 & $13.90(\mathrm{R})$ & 248 & 0.404708 & $1.87 \mathrm{E}-40$ & 0.507660 & 6 \\
\hline 2MASS J20292467+6029444 & 202924.67 & +602944.4 & $16.20(\mathrm{R})$ & 180 & - & - & 0.451130 & 5 \\
\hline NN Aqr & 214354.00 & -001341.0 & $13.47(\mathrm{R})$ & 255 & 0.306771 & $9.86 \mathrm{E}-36$ & 0.306780 & 6 \\
\hline V441 Lac & 220937.53 & +523416.0 & $12.20(\mathrm{R})$ & 2075 & 0.308915 & $7.41 \mathrm{E}-219$ & 0.308911 & 7 \\
\hline PP Lac & 224238.66 & +532502.9 & 11.68 & 88 & 0.401103 & $1.46 \mathrm{E}-09$ & 0.401163 & 6 \\
\hline V357 Peg & 234535.07 & +252818.9 & $9.39(\mathrm{~B})$ & 342 & 0.578422 & $3.35 \mathrm{E}-56$ & 0.578451 & 14 \\
\hline $\mathrm{Cl}^{*} \mathrm{NGC} 7789$ XZD 3 & 235653.75 & +562149.1 & 13.99 & 976 & 0.345073 & $9.11 \mathrm{E}-76$ & 0.344700 & 11 \\
\hline Cl* NGC 7789 XZD 10 & 235824.38 & +570914.7 & $13.88(\mathrm{R})$ & 1380 & 0.830513 & $5.76 \mathrm{E}-15$ & 0.844200 & 11 \\
\hline Cl* NGC 7789 XZD 7 & 235902.75 & +570537.1 & 16.48 & 430 & 0.395180 & $4.32 \mathrm{E}-15$ & 0.395600 & 11 \\
\hline
\end{tabular}

Notes. Targets are in RA order. Periods given in bold are discusses in the text.

${ }^{a}$ References. (1) Gettel, Geske, \& McKay (2006); (2) Pigulski et al. (2009); (3) Devor et al. (2008); (4) Akerlof et al. (2000); (5) Kafka et al. (2004); (6) Samus et al. (2008); (7) Kreiner (2004); (8) Zhang et al. (2004); (9) Vanko \& Pribulla (2001); (10) Virnina (2011); (11) Xin, Zhang, \& Deng (2002); (12) Hoffmann \& Meinunger (1983); (13) Kolesnikova et al. (2008); (14) Rucinski et al. (2008); (15) Khruslov (2006); (16) Kuzmin (2008); (17) Özkardeş \& Erdem (2007); (18) Kinman, Mahaffey \& Wirtanen (1982).

This common effect might have also caused extra scattering in the light curves of the systems we analyzed.

We extended our work to obtain Fourier coefficients by decomposing the light curves of $40 \mathrm{~W}$ UMa-type systems. Although the period determination was successful for the systems Cl* NGC 7789 XZD 10 and V473 And, the Fourier series fitting process has failed probably due to the large scatter in their light curves. Therefore, 38 systems were studied by this method. Figure 1 shows a representative Fourier fit for the system 2MASS J11403001+7111021.
Rucinski (1997b) has shown that a boundary line between the $a_{2}$ and $a_{4}$ coefficients, which are always negative, as $a_{4}=a_{2}\left(0.125-a_{2}\right)$ can be used to distinguish binary systems showing $\beta$ Lyr (EB) or W UMa (EW) type light curves from detached (EA) binaries. They also took samples of pulsating stars to show that these systems can be easily distinguished from the eclipsing binaries by falling on the lower part (positive $a_{4}$ values) of the $a_{2}-a_{4}$ diagram (see Figure 4 of Rucinski 1997b). Results of this study for the 38 systems are listed in Table 2 and shown graphically 
Table 2. Selected W UMa-Type Binaries from the ROTSE-IIId Database with Calculated Fourier Coefficients and Corresponding System Parameters

\begin{tabular}{|c|c|c|c|c|c|c|c|c|c|c|c|}
\hline Name & $a_{1}$ & $a_{2}$ & $a_{4}$ & $f$ & $i$ & $q$ & $\mathrm{f}_{1}$ & $\mathrm{i}_{1}$ & $\mathrm{q}_{1}$ & $L 3$ & $\operatorname{Ref}^{a}$ \\
\hline V959 Cas & $-0.008(4)$ & $-0.166(4)$ & $-0.032(4)$ & 0.4 & 77.5 & 0.25 & - & - & - & - & - \\
\hline V472 And & $-0.012(5)$ & $-0.099(5)$ & $-0.024(5)$ & 0.1 & 65.0 & 0.2 & - & - & - & - & - \\
\hline V473 And & - & - & - & - & - & - & - & - & - & - & - \\
\hline V755 Cep & $+0.001(4)$ & $-0.131(4)$ & $-0.012(4)$ & 0.4 & 60.0 & 0.95 & - & - & - & - & - \\
\hline V490 And & $-0.008(6)$ & $-0.112(6)$ & $-0.006(6)$ & 0.9 & 47.5 & 0.75 & - & - & - & - & - \\
\hline ES Cep & $+0.009(6)$ & $-0.118(6)$ & $-0.024(6)$ & 0.0 & 65.0 & 0.65 & - & $70.99,70$ & $0.782,0.4$ & - & 11,12 \\
\hline V790 Cep & $-0.005(1)$ & $-0.032(1)$ & $-0.003(1)$ & 0.0 & 40.0 & 0.30 & - & - & - & - & - \\
\hline V793 Cep & $-0.015(9)$ & $-0.102(9)$ & $-0.021(9)$ & 0.0 & 80.0 & 0.10 & - & - & - & - & - \\
\hline GR Psc & $+0.013(6)$ & $-0.194(7)$ & $-0.014(6)$ & 0.7 & 67.5 & 1.00 & - & - & - & - & - \\
\hline V1107 Cas & $-0.014(5)$ & $-0.156(5)$ & $-0.043(5)$ & 0.0 & 80.0 & 0.25 & - & - & - & - & - \\
\hline DN Cam & $+0.001(9)$ & $-0.161(9)$ & $-0.035(9)$ & 0.2 & 70.0 & 0.85 & $0.5,0.33$ & $71.9,73.1$ & $0.421,0.442$ & - & 3,4 \\
\hline V415 Cam & $-0.002(4)$ & $-0.148(4)$ & $-0.022(4)$ & 0.3 & 77.5 & 0.20 & - & - & - & - & - \\
\hline AH Gem & $+0.004(7)$ & $-0.118(7)$ & $-0.017(7)$ & 0.5 & 55.0 & 0.90 & - & - & - & - & - \\
\hline V455 Mon & $+0.014(9)$ & $-0.230(9)$ & $-0.047(9)$ & 0.5 & 80.0 & 0.85 & - & 83.5 & 0.9 & - & 12 \\
\hline CZ CMi & $+0.868(2)$ & $-0.015(6)$ & $-0.002(6)$ & 0.4 & 65.0 & 0.70 & 0.295 & 79.0 & 0.24 & $\begin{array}{c}B=0.61 \\
V=050\end{array}$ & 2 \\
\hline XX Lyn & $-0.027(8)$ & $-0.167(8)$ & $-0.005(9)$ & 0.8 & 70.0 & 0.30 & - & - & - & - & - \\
\hline $\mathrm{AH} \mathrm{Cnc}$ & $+0.000(4)$ & $-0.144(4)$ & $-0.010(4)$ & 1.0 & 67.5 & 0.20 & 0.56 & 88.2 & 0.168 & - & 1 \\
\hline RT LMi & $+0.004(4)$ & $-0.199(3)$ & $-0.045(4)$ & 0.3 & 77.5 & 0.60 & $0.28,0.282$ & $83.2,84.1$ & $0.382,0.378$ & - & 6,7 \\
\hline 2MASS J11403001+7111021 & $-0.002(2)$ & $-0.129(2)$ & $-0.018(2)$ & 0.4 & 67.5 & 0.25 & - & - & - & - & - \\
\hline 2MASS J11483649+7107507 & $-0.015(3)$ & $-0.164(3)$ & $-0.035(3)$ & 0.2 & 75.0 & 0.35 & - & - & & & \\
\hline GQ Boo & $-0.002(5)$ & $-0.116(5)$ & $-0.013(5)$ & 0.3 & 67.5 & 0.20 & - & - & - & - & - \\
\hline GR Boo & $-0.011(4)$ & $-0.135(5)$ & $-0.022(5)$ & 0.3 & 72.5 & 0.20 & - & - & - & - & - \\
\hline V829 Her & $-0.010(2)$ & $-0.089(2)$ & $-0.008(2)$ & 0.8 & 42.5 & 0.85 & $0.19-0.27$ & $55.0-57.7$ & 0.435 & - & 10 \\
\hline V2203 Oph & $-0.022(2)$ & $-0.128(2)$ & $-0.022(2)$ & 0.1 & 65.0 & 1.00 & - & - & - & - & - \\
\hline V591 Lyr & $-0.011(5)$ & $-0.165(4)$ & $-0.036(4)$ & 0.2 & 75.0 & 0.35 & - & - & - & - & - \\
\hline V574 Lyr & $+0.006(9)$ & $-0.169(9)$ & $-0.048(8)$ & 0.0 & 75.0 & 0.60 & - & - & - & - & - \\
\hline 2MASS J18413909-0044433 & $-0.016(9)$ & $-0.115(9)$ & $-0.011(9)$ & 0.9 & 47.5 & 0.85 & - & - & - & - & - \\
\hline 2MASS J18562000+4556486 & $+0.022(9)$ & $-0.174(9)$ & $-0.030(9)$ & 0.4 & 70.0 & 0.80 & - & - & - & - & - \\
\hline 2MASS J19062202+4338096 & $-0.014(4)$ & $-0.218(4)$ & $-0.055(4)$ & 0.3 & 82.5 & 0.55 & - & - & - & - & - \\
\hline ROTSE1 J190830.01+433601.5 & $-0.003(1)$ & $-0.064(1)$ & $-0.017(1)$ & 0.1 & 70.0 & 0.05 & - & - & - & - & - \\
\hline 2MASS J19180543+4441153 & $+0.002(4)$ & $-0.165(4)$ & $-0.029(4)$ & 0.4 & 67.5 & 1.00 & - & - & - & - & - \\
\hline V2364 Cyg & $-0.003(5)$ & $-0.170(5)$ & $-0.020(5)$ & 0.5 & 77.5 & 0.25 & - & 81.8 & 0.306 & - & 8 \\
\hline V981 Cyg & $-0.018(4)$ & $-0.190(4)$ & $-0.037(4)$ & 0.3 & 77.5 & 0.45 & - & 74.0 & 0.160 & - & 12 \\
\hline NN Aqr & $-0.008(6)$ & $-0.174(6)$ & $-0.055(6)$ & 0.0 & 75.0 & 0.8 & - & - & - & - & - \\
\hline V441 Lac & $-0.013(2)$ & $-0.077(2)$ & $-0.017(2)$ & 0.0 & 57.5 & 0.35 & - & - & - & - & - \\
\hline PP Lac & $-0.020(4)$ & $-0.216(4)$ & $-0.049(4)$ & 0.3 & 80.0 & 0.70 & 0.239 & 83.9 & 0.43 & - & 5 \\
\hline V357 Peg & $-0.007(3)$ & $-0.161(3)$ & $-0.020(3)$ & 0.8 & 60.0 & 0.80 & 0.10 & 73.23 & 0.401 & - & 9 \\
\hline $\mathrm{Cl}^{*} \mathrm{NGC} 7789$ XZD 3 & $-0.018(6)$ & $-0.141(6)$ & $-0.039(6)$ & 0.0 & 70.0 & 0.50 & - & - & - & - & - \\
\hline $\mathrm{Cl}^{*} \mathrm{NGC} 7789$ XZD 10 & - & - & - & - & - & - & - & - & - & - & - \\
\hline $\mathrm{Cl}^{*}$ NGC 7789 XZD 7 & $-0.014(9)$ & $-0.156(9)$ & $-0.018(9)$ & 0.6 & 62.5 & 0.80 & - & - & - & - & - \\
\hline
\end{tabular}

Notes. The numbers in the parentheses represent standard error of the last digit. Three system parameters, $f_{l}, i_{l}$, and $q_{l}$, if found in the literature are given between columns 7 and 10. Two systems that have failed in the Fourier series fitting process are discussed in the text.

${ }^{a}$ References. (1) Yakut et al. (2009); (2) Yang et al. (2005); (3) Vanko \& Pribulla (2001); (4) Baran et al. (2004); (5) Qian, Zhu, \& Boonruksar (2005); (6) Zola et al. (2010); (7) Qian et al. (2008); (8) Nelson et al. (2002); (9) Deb \& Singh (2011); (10) Erdem \& Özkardeş (2006); (11) Branly et al. (1996); (12) Svechnikov \& Kuznetsova (1990).

in Figure 2. Although five systems, NN Aqr, V472 And, Cl* NGC 7789 XZD 3, ROTSE1J190830.01+433601.5, and V441 Lac, are located inside the detached system area, they are very close to the boundary. This indicates that the candidates are contact or semi-detached systems.

There is another correlation proposed by Rucinski (1997a) to separate EB systems from EW systems on the $a_{1}$ versus $a_{2}$ plane in which the $a_{1}=-0.02$ line distinguishes EB systems from EW systems. This criterion is based on the fact that most of the EW-type systems show small differences in eclipse depths by the usage of Fourier coefficient $a_{1}$, which is related to the depths of the eclipses. Of the $38 \mathrm{EW}$-type binaries that have been analysed, only 2 systems, XX Lyn and V2203 Oph, seem to fall into the EB-type region. However, they are too close to the boundary for us to make any decisions when their error bars are also taken into account. This also indicates that all the 38 candidates are well represented to be genuine $\mathrm{W}$ UMa-type binaries on the basis of this second criterion (see Figure 3). 


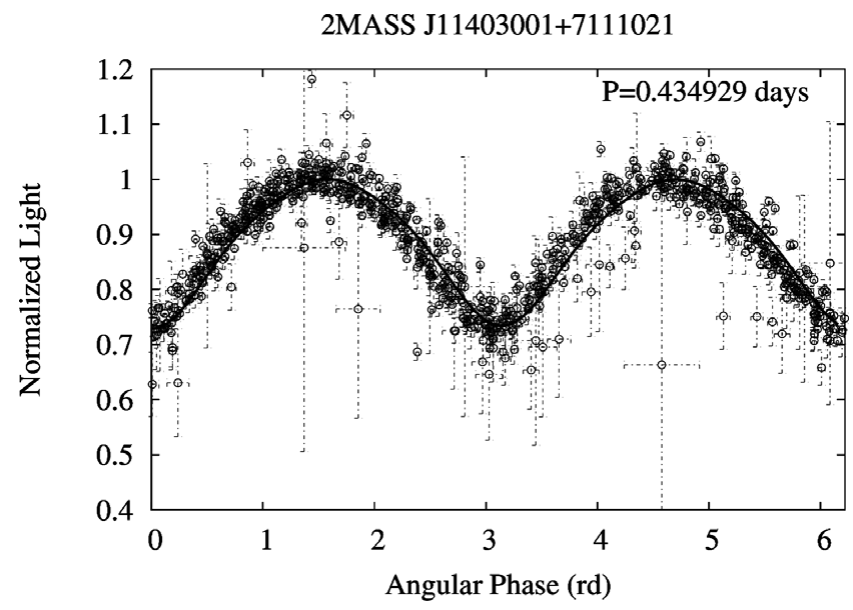

Figure 1. A sample of the folded and unity normalised light curve of 2MASS J11403001+7111021, observed by the ROTSE-IIId telescope between 2004 and 2009, is presented. Period based on the L-S algorithm and theoretical curve (solid line) obtained by Fourier series fitting are also shown in the figure.

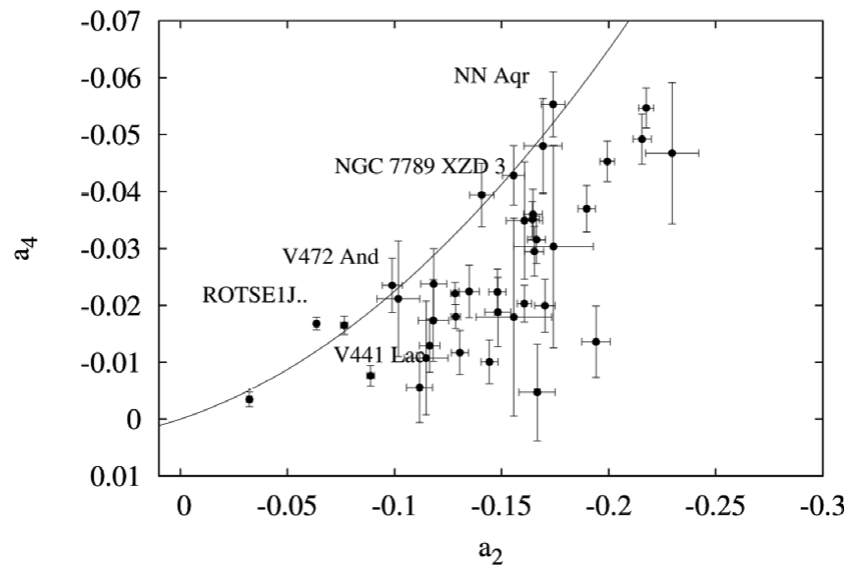

Figure 2. Distribution of the $38 \mathrm{~W}$ UMa-type systems on the $a_{2}-a_{4}$ plane. There are no points below $a_{4}=0$ which would have been an indicator of a pulsating system (Rucinski 1997b). All points that lie below the boundary line shown by the solid curve are either EW- or EB-type binary.

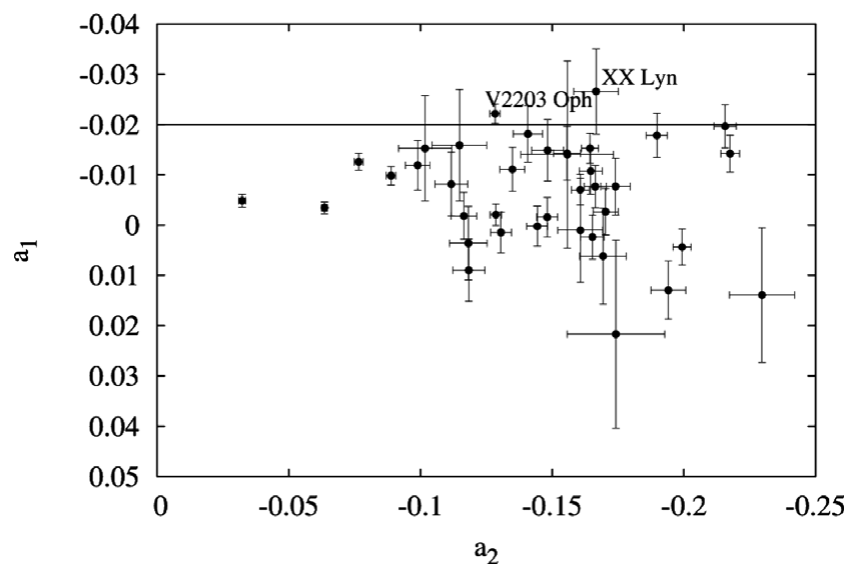

Figure 3. Distribution of the $38 \mathrm{~W}$ UMa-type systems on the $a_{1}-a_{2}$ plane. Fourier coefficient $a_{1}$ is related to the difference between the depths of the eclipses.
A few geometrical parameters, such as $f, i$, and $q$, can also be estimated using Fourier coefficients. The table of coefficients on distinct inclination angles and mass ratios for three set of fill-out parameters, $f=0,0.5$, and 1.0, was accessed from Rucinski's personal Web site. As suggested by Rucinski (1993), the $a_{2}$ and $a_{4}$ coefficients were primarily used in estimating $f$ values, and then only $a_{2}$, which is highly correlated with the depth of the eclipse, was used to obtain the $i$ and $q$ parameters. Calculated Fourier coefficients $a_{1}$, $a_{2}$, and $a_{4}$, and corresponding geometrical parameters $f, i$, and $q$ with literature information, are given in Table 2 for 38 systems. Values of 27 systems are the first approximations.

\section{CONCLUSION}

Fourier series fitting in recognizing the types of variability was studied and tested using the light curves generated from the ROTSE-IIId archive data for a total of $45 \mathrm{~W}$ UMatype sample systems. Among the dataset, some systems such as the three that failed in period determination, two with lower brightness than the ROTSE-IIId limiting magnitude, and two that have uncertain Fourier fitting, were excluded from further analysis. Therefore, Fourier series fitting and estimates on preliminary system parameters of the remaining 38 systems were performed. Results show that using combinations of the Fourier coefficient as a filtering technique is very successful in identifying the EW-type systems. However, in Table 2, there are some discrepancies in the system parameters $f, i$, and $q$, between exact solutions given in the literature and those predicted by this method. This unconformity may be attributed to scattered or distorted light curves by an instrumental effect, any third light contamination, O'Connell effect, or spot activity; i.e. see Yang et al. (2005) for a possible third light in CZ CMi, Zola et al. (2010) and Qian, He, \& Xiang (2008) for light-curve asymmetries in RT LMi, Özkardeş \& Erdem (2007) for the O'Connell effect and spot activity in V829 Her, and Nelson et al. (2002) for spot activity in V2364 Cyg. In addition, this technique is not sensitive to recognising the systems with total eclipses as reported by Rucinski (1993). Such a system, AH Cnc, exhibits total eclipses in the light curves of Yakut et al. (2009). However, our light curve of $\mathrm{AH} \mathrm{Cnc}$ does not show any sign of totality. This may be the cause of the difference in the system parameters of $\mathrm{AH} \mathrm{Cnc}$ between the light-curve solution and Fourier estimations in Table 2. If the light curves are contaminated by all these distorting effects, the reliability of the method decreases. Another difficulty encountered in defining the fill-out factor $f$ emerges in the lower, i.e. near-zero, part of the $a_{2}$ versus $a_{4}$ plane where all values for each fill-out are mixed. However, the technique is still a very powerful tool for classifying huge amounts of photometric data gathered by automated sky surveys for a few decades. In the future, the plan is to apply this Fourier series fitting technique to identify all eclipsing W UMa-type systems in the full dataset of the ROTSE-IIId archive. 


\section{ACKNOWLEDGMENTS}

This project utilises data obtained by the Robotic Optical Transient Search Experiment (ROTSE). ROTSE is a collaboration of the Lawrence Livermore National Laboratory, the Los Alamos National Laboratory, and the University of Michigan (http://www.rotse.net).

All observations were made with the ROTSE-IIId telescope and the archival data of ROTSE-IIId obtained at the TÜBİTAK (Turkish Scientific and Research Council) National Observatory (TUG); therefore, we thank the ROTSE-III Collaboration and TUG for the optical and archival facilities (TUG-ROTSE-IIId projects of Turkish observers).

We thank the anonymous referee for a positive review of the manuscript that has improved its content. We also thank Prof. Dr. Ü. Kızıloğlu for consultation, suggestions, and help.

This study was supported by TÜBİTAK with the project TBAG$108 \mathrm{~T} 475$

This research has made use of the SIMBAD database, operated at CDS, Strasbourg, France, and the CDSCLIENT tool located at CDS and NASA Astrophysics Data System Bibliographic Services.

\section{REFERENCES}

Akerlof, C., et al. 2000, AJ, 119, 1901

Akerlof, C. W., et al. 2003, PASP, 115, 132

Awadalla, N. S. 1988, Ap\&SS, 140, 137

Awadalla, N. S. 1994, A\&A, 289, 137

Baran, A., Zola, S., Rucinski, S. M., Kreiner, J. M., Siwak, M., \& Drozdz, M. 2004, AcA, 54, 195

Bertin, E., \& Arnouts, S. 1996, A\&AS, 117, 393

Binnendijk, L. 1970, VA, 12, 217

Binnendijk, L. 1977, VA, 21, 359

Borucki, W. J., et al. 2010, Sci, 327, 977

Branly, R. M., Athauda, R. I., Fillingim, M. O., \& van Hamme, W. 1996, Ap\&SS, 235, 149

Deb, S., \& Singh, H. P. 2011, MNRAS, 412, 1787

Derman, E., Demircan, O., \& Selam, S. 1991, A\&AS, 90, 301

Devor, J., Charbonneau, D., O’Donovan, F. T., Mandushev, G., \& Torres, G. 2008, AJ, 135, 850

Eggleton, P. P. 1996, in ASP Conf. Ser. Vol. 90, The Origins, Evolution, and Destinies of Binary Stars in Clusters, ed. E. F. Milone \& J.-C. Mermilliod (San Francisco, CA: ASP), 257

Erdem, A., \& Özkardeş, B. 2006, NewA, 12, 192

Foster, G. 1995, AJ, 109, 1889

Gettel, S. J., Geske, M. T., \& McKay, T. A. 2006, AJ, 131, 621

Güçsav, B. B., Yeşilyaprak, C., Yerli, S. K., Aksaker, N., Kızıloğlu, U., Çoker, D., Dikicioğlu, E., \& Aydın, M. E. 2012, ExA, 33, 197

Hoffmann, M., \& Meinunger, L. 1983, IBVS, 2343, 1

Kafka, S., Gibbs II, D. G., Henden, A. A., \& Honeycutt, R. K. 2004, AJ, 127,1622

Kähler, H. 2004, A\&A, 414, 317

Karimie, M. T. 1983, Ap\&SS, 92, 53

Khruslov, A. V. 2006, PZP, 6, 11
Kinman, T. D., Mahaffey, C. T., \& Wirtanen, C. A. 1982, AJ, 87, 314

Kolesnikova, D. M., Sat, L. A., Sokolovsky, K. V., Antipin, S. V., \& Samus, N. N. 2008, AcA, 58, 279

Kreiner, J. M. 2004, AcA, 54, 207

Kuzmin, M. L. 2008, PZP, 8, 15

Li, L., Zhang, F., Han, Z., Jiang, D., \& Jiang, T. 2008, in IAU Symp. 252, Structure and Evolution of W UMa-Type Systems, ed. L. Deng \& K. L. Chan (Cambridge: Cambridge Univ. Press), 423

Lomb, N. R. 1976, Ap\&SS, 39, 447

Lucy, L. B. 1968, ApJ, 151, 1123

Mas-Hesse, J. M., Giménez, A., Domingo, A., Rísquez, D., Caballero, M. D., Gutiérrez, R., Solano, E., the OMC Team 2004, in ESA Special Publication Vol. 552, 5th INTEGRAL Workshop on the INTEGRAL Universe, ed. V. Schoenfelder, G. Lichti, \& C. Winkler (Noordwijk: ESA), 729

Monet, D. G. 1998, AAS Abstracts, 30, 120.03

Nelson, R. H., Robb, R. M., Kaiser, D. H., \& Billings, G. B. 2002, IBVS, 5285, 1

Özkardeş, B., \& Erdem, A. 2007, in ASP Conf. Ser. Vol. 370, Photometric Analysis of the Contact Binary System V829 Hercules, ed. O. Demircan, S. O. Selam, \& B. Albayrak (San Francisco, CA: ASP), 237

Pigulski, A., Pojmański, G., Pilecki, B., \& Szczygieł, D. M. 2009, AcA, 59, 33

Qian, S.-B., He, J.-J., \& Xiang, F.-Y. 2008, PASJ, 60, 77

Qian, S.-B., Zhu, L.-Y., \& Boonruksar, S. 2005, NewA, 11, 52

Rucinski, S. M. 1973, AcA, 23, 79

Rucinski, S. M. 1993, PASP, 105, 1433

Rucinski, S. M. 1997a, AJ, 113, 1112

Rucinski, S. M. 1997b, AJ, 113, 407

Rucinski, S. M., et al. 2008, AJ, 136, 586

Samus, N. N., et al. 2008, VizieR Online Data Catalog, 1, 2025

Scargle, J. D. 1982, ApJ, 263, 835

Schwarzenberg-Czerny, A. 1996, ApJ, 460, L107

Selam, S. O. 2004, A\&A, 416, 1097

Svechnikov, M. A., \& Kuznetsova, E. F. 1990, Catalog of Approximate Photometric and Absolute Elements of Eclipsing Variable Stars (Sverdlovsk: Ural Univ. Press)

Udalski, A., Szymanski, M., Kaluzny, J., Kubiak, M., \& Mateo, M. 1992, AcA, 42, 253

Udalski, A., Szymanski, M., Kaluzny, J., Kubiak, M., Mateo, M., \& Krzeminski, W. 1994, ApJ, 426, L69

Vanko, M., \& Pribulla, T. 2001, IBVS, 5200, 1

Virnina, N. A. 2011, OEJV, 133, 1

Xin, Y., Zhang, X.-B., \& Deng, L.-C. 2002, ChJAA, 2, 481

Yakut, K., et al. 2009, A\&A, 503, 165

Yang, Y.-G., Qian, S.-B., Zhu, L.-Y., He, J.-J., \& Yuan, J.-Z. 2005, PASJ, 57, 983

Zhang, X. B., Deng, L., Zhou, X., \& Xin, Y. 2004, MNRAS, 355, 1369

Zola, S., Gazeas, K., Kreiner, J. M., Ogloza, W., Siwak, M., Koziel-Wierzbowska, D., \& Winiarski, M. 2010, MNRAS, 408, 464 